\title{
lodine/TBHP
}

\section{Gandhesiri Satish}

School of Chemistry, Bharathidasan University, Tiruchirappalli - 620 024, Tamilnadu, India

sathish.gandhesiri@gmail.com

Published online: 11.06 .2015

DOI: 10.1055/s-0034-1380899; Art ID: st-2015-v0523-v

\section{Introduction}

In recent times, several advancements were made in using catalytic amounts of iodine in combination with tertbutyl hydroperoxide (TBHP) as co-oxidant. The increased utility is mainly due to its inexpensive, environmentally be-
Gandhesiri Satish was born in Kannepalle, Telangana, India in 1986. He obtained his M.Sc. in organic chemistry (2009) from Kakatiya University, India. He qualified for CSIR (JRF) and GATE and currently he is a doctoral student under the supervision of Prof. Andivelu llangovan. His research interest mainly focuses on the development of $\mathrm{C}-\mathrm{C}$ and $\mathrm{C}$-heteroatom bond formation for bioactive compounds via $\mathrm{C}-\mathrm{H}$ activation strategy.

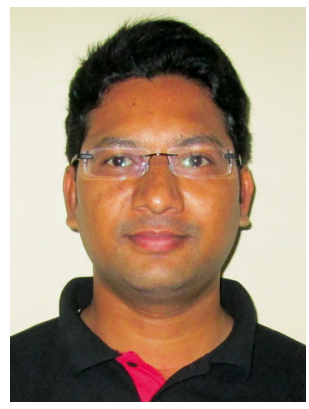

Table 1 Use of lodine-TBHP

(A) Jiang et al. reported an $\mathrm{I}_{2} /$ TBHP mediated domino oxidative cyclization for the one-pot synthesis of polysubstituted oxazoles from readily available styrenes and benzylamines under mild conditions. ${ }^{3}$

(B) Zhang et al. demonstrated the synthesis of 2-phenylquinazolines in good to excellent yields via tandem $\mathrm{sp}^{3} \mathrm{C}-\mathrm{H}$ functionalization of 2-amino benzophenones and benzylic amines. ${ }^{4}$

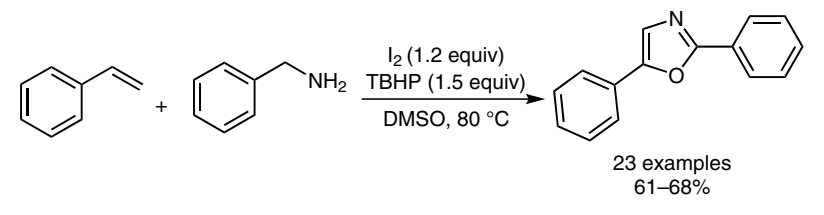

nign nature, good efficiency and compatibility to work in place of rare or toxic heavy metal oxidants. ${ }^{1}$ The $\mathrm{I}_{2} / \mathrm{TBHP}$ catalytic system works efficiently for numerous $\mathrm{C}-\mathrm{C}$ and $\mathrm{C}-$ $\mathrm{X}(\mathrm{X}=$ heteroatom $)$ bond-forming organic transformations under mild reaction conditions to offer the desired products in excellent yields. ${ }^{2}$

(C) Manjunath and Prabhu reported a metal-free catalytic route to 2 -aminobenzoxazoles by amination of benzoxazoles via $\mathrm{C}-\mathrm{H}$ bond activation of primary or secondary amines. Further the methodology was demonstrated to synthesize therapeutically active benzoxazoles. $^{5}$
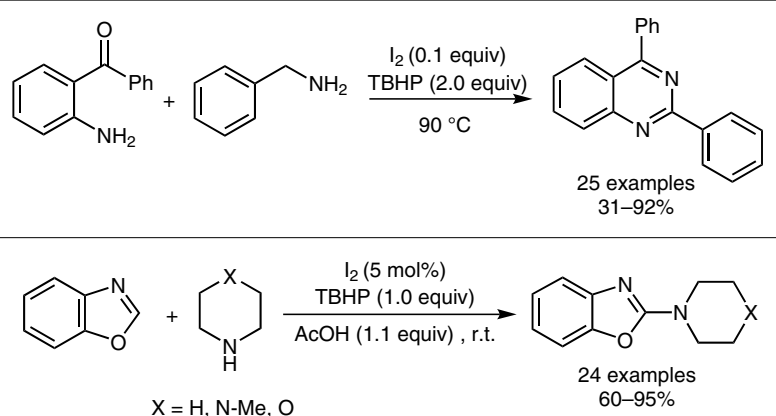

(D) An $\mathrm{I}_{2} / \mathrm{TBHP}$ catalyzed first efficient and direct synthesis of tertiary amides from alcohols and dimethylformamide has been developed. This transition-metal-free protocol provides a practical synthetic tool for the construction of $N, N$-dimethyl-substituted amides. ${ }^{6}$

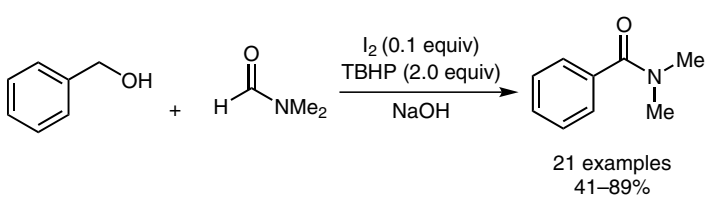


(E) A metal-free oxidative coupling of methyl ketones and primary or secondary amines to $\alpha$-keto amides was developed by Wan and his group. Four types of intermediates, $\alpha$-iodo ketones, $\alpha$-amino ketones, iminium intermediates and $\alpha$-hydroxy amines were identified through a series of control experiments. The atom-economic methodology can be scaled-up, tolerates a variety of functional groups, and is operationally simple. ${ }^{7}$

(F) A novel $\mathrm{I}_{2} /$ TBHP catalyzed selective 2-arylsulfonylation of indoles was demonstrated. Various substituted 2-arylsulfonyl indoles were obtained in good to excellent yields in one pot. The direct sulfonylation reaction occurred selectively at $\mathrm{C}-2$ position of the indole ring and only a catalytic amount of iodine acted as an efficient promoter. This method is a novel alternative approach for the synthesis of biologically important hetero diaryl sulfones from sodium sulfinates. ${ }^{8}$

(G) Recently, we have developed an $\mathrm{I}_{2} / \mathrm{TBHP}$ mediated synthesis of isatin and iodoisatin from 2 -aminoacetophenone via intramoleular oxidative amidation of the $\mathrm{sp}^{3} \mathrm{C}-\mathrm{H}$ bond. The reaction proceeds through sequential iodination, Kornblum oxidation, and amidation in one pot. The stoichiometric amount of $\mathrm{I}_{2}$ plays a significant role in delivering iodoisatin exclusively in high yields. ${ }^{9}$
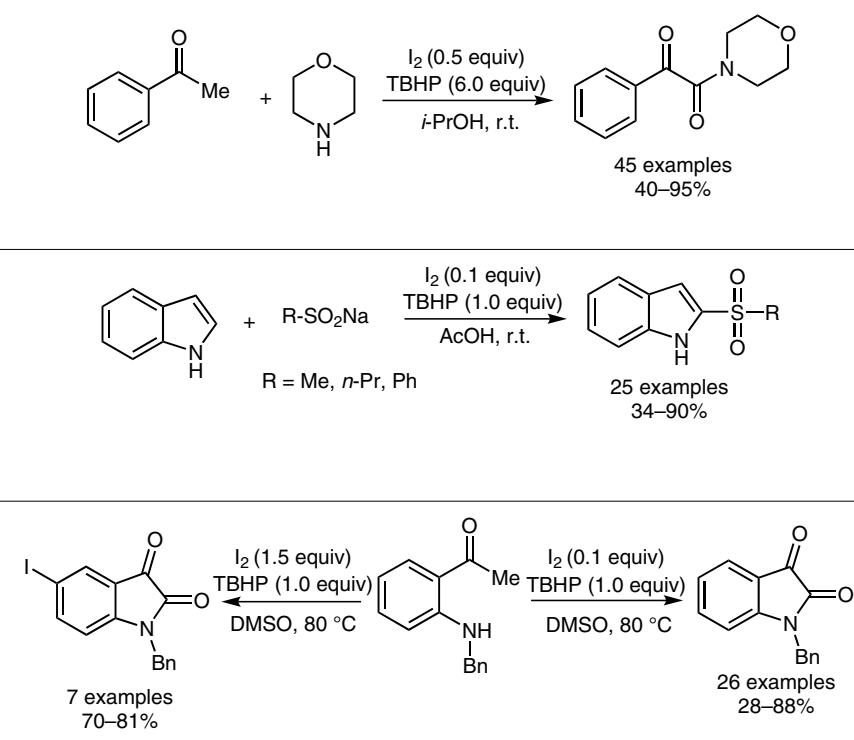

(H) Ji's research group reported an $\mathrm{I}_{2} /$ TBHP mediated oxidation of commercially available indoles to isatins in moderate to good yields. ${ }^{10}$

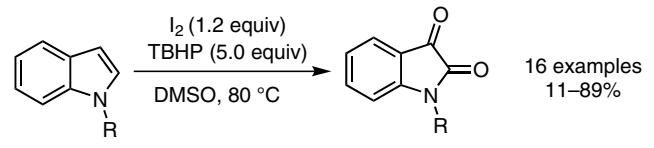

\section{References}

(1) (a) Finkbeiner, P.; Nachtsheim, B. J. Synthesis 2013, 979. (b) Kirihara, M.; Asai, Y.; Ogawa, S.; Noguchi, T.; Hatano, A.; Hirai, Y. Synthesis 2007, 3286.

(2) (a) Hummel, S.; Kirsch, S. F. Beilstein J. Org. Chem. 2011, 7, 847. (b) Zhao, Q.; Miao, T.; Zhang, X.; Zhou, W.; Wang, L. Org. Biomol. Chem. 2013, 11, 1867.

(3) Jiang, H.; Huang, H.; Cao, H.; Qi, C. Org. Lett. 2010, 12, 5561.

(4) Zhang, J.; Zhu, D.; Yu, C.; Wan, C.; Wang, Z. Org. Lett. 2010, 12, 2841.
(5) Manjunath, L.; Prabhu, K. R. J. Org. Chem. 2011, 76, 7938.

(6) Xu, K.; Hu, Y.; Zhang, S.; Zha, Z.; Wang, Z. Chem. Eur. J. 2012, 18, 9793.

(7) Wei, W.; Shao, Y.; Hu, H.; Zhang, F.; Zhang, C.; Xu, Y.; Wan, X. J. Org. Chem. 2012, 77, 7157.

(8) Xiao, F.; Chen, H.; Xie, H.; Chen, S.; Yang, L.; Deng, G.-J. Org. Lett. 2014, 16, 50.

(9) Ilangovan, A.; Satish, G. J. Org. Chem. 2014, 79, 4984.

(10) Zi, Y.; Cai, Z.-J.; Wang, S.-Y.; Ji, S.-J. Org. Lett. 2014, 16, 3094. 